\title{
Multipath Fading Compensation Techniques Using Pilot and Data Symbols in Mobile Radio Channels
}

\author{
H. K. Lau and S. W. Cheung \\ Communications Research Group \\ Department of Electrical and Electronic Engineering \\ The University of Hong Kong \\ Pokfulam Road \\ HONG KONG
}

\begin{abstract}
The paper studies techniques for transmitting a 16-ary quadrature-amplitude modulated (16QAM) signal in different mobile radio environments. Conventional multipath fading compensation techniques using only the pilot symbols, incorporated with zeroth-order and firstorder prediction methods, are studied. Novel techniques making use both the pilot symbols and the data symbols, together with the improved zeroth-order and first-order prediction methods, are proposed. A series of intensive Monte Carlo simulations has been carried out on these techniques and the results have shown that, at high signal-tonoise ratios (SNRs), the bit-error rate (BER) performance can be reduced by a factor of about 46 and 2 using the improved zeroth-order and first-order prediction respectively, relative to those obtained using the pilot symbols only.
\end{abstract}

\section{Introduction}

It is envisaged that Personal Communications Services (PCS) will become an important part of the future telecommunication services. Digital cellular technologies such as Digital Advanced Mobile Phone Service (DAMPS) in the United States, Pan-European Group Sp'eciale Mobile system (GSM) in Europe, and Japanese Digital Cellular system (JDC) in Japan are being developed. The range and applications of cellular services are undergoing a rapid evolution. However, due to the severe multipath fading distortions introduced into the signals by the mobile radio channels, signal constellations for the existing digital cellular systems are limited to 2 bits per symbol or less. To cope the ever-increasing demand for radio spectrum, multilevel signals are expected to be used for mobile communication systems in the future.

Recently, the pilot symbol-aided techniques have been shown to be suitable for transmitting multilevel signals in mobile radio environments [1-7]. In a pilot symbol-aided system, the data-symbol sequence is divided into frames of symbols for transmission. A known pilot symbol is inserted at the beginning of each frame. These pilot symbols are used for multipath fading compensation at the receiver. Conventional approaches require the receivers to store up the data symbols before fading correction is applied [2-5]. A technique uses extrapolation prediction that requires no storage of the previous data symbols has been proposed and analyzed in [7].

This paper, on the other hand, proposes the improved multipath fading compensation (estimation and correction) techniques which are the extension of the existing pilot symbolaided schemes. The techniques use the distortion information in both the pilot symbols and data symbols for multipath fading compensation. Monte Carlo simulations have been carried out to investigate the effects of the proposed techniques on BER performance of 16QAM signal, in the Rayleigh fading channels. Results have shown that significant improvements in the BER performance can be obtained by the proposed techniques, relative to those only using the pilot symbols.

\section{System Overview}

The baseband equivalent model of the data-transmission system considered in the study is shown in Figure 1.

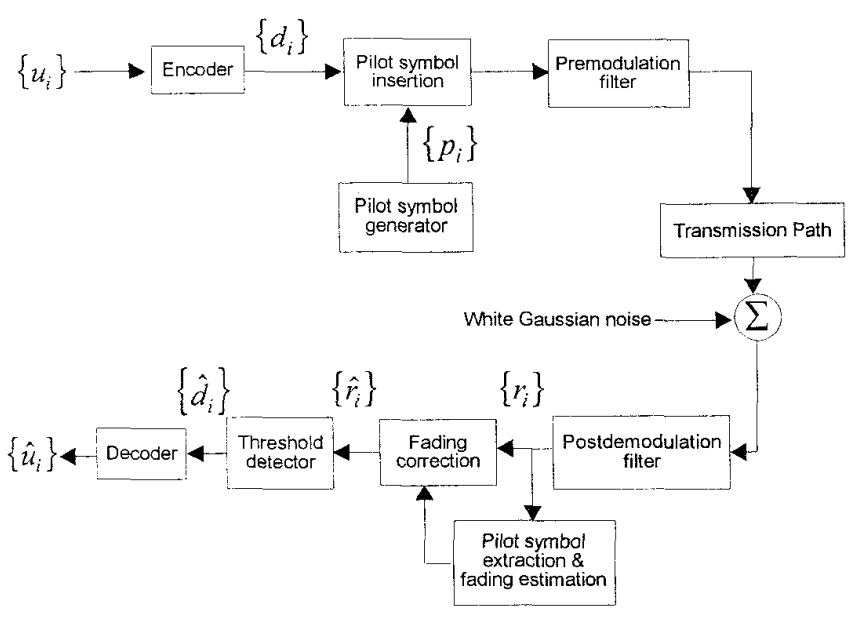

Figure 1 Model of System 
When the encoder has received the binary digits $\left\{u_{i}\right\}$, it maps these signals into a data symbol $d_{i}$ according to the 16QAM signal constellation. For every $(L-1)$-data symbols, a pilot symbol $p_{i}$ is inserted to form an $L$-symbol frame. To minimize the degradation due to additive white Gaussian noise, the pilot symbols $\left\{p_{i}\right\}$ are pseudo randomly chosen from those signal vectors with the largest energy level in the constellation [7]. Since the receiver has the prior knowledge of the $\left\{p_{i}\right\}$, it can estimate the multipath fading distortion introduced in the transmission path and make the appropriate correction to the received signal. The threshold detector and decoder (Figure 1) then operate on the corrected data symbols $\left\{\hat{r}_{i}\right\}$ to produce the binary data $\left\{\hat{u}_{i}\right\}$ at the output. The transmission path in Figure 1 is a time-varying linear baseband channel that introduces Rayleigh fading [8].

\section{Multipath Fading Compensation}

\section{Zeroth-Order and First-Order Predictions}

The frame structure of the transmitted symbol sequence is shown in Figure 2.

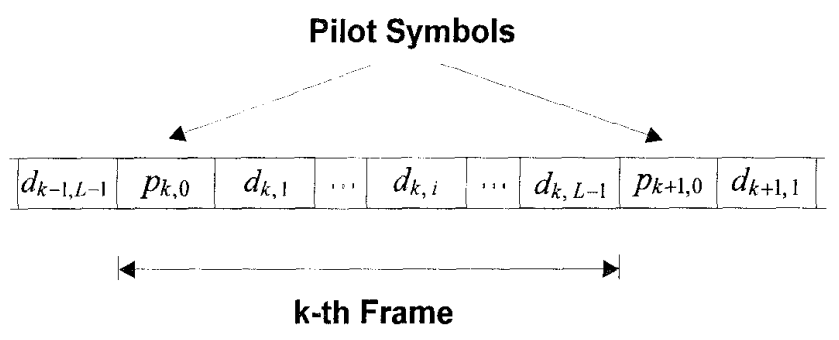

Figure 2 Frame Structure of Transmitted Symbol Sequence

The sample signal at the $i$-th position of the $k$-th received frame can be written as

$$
r_{k, i}=q_{k, i} y_{k, i}+w_{k, i}
$$

where $q_{k, i}$ is either a pilot symbol or a data symbol,

$y_{k, i}$ is the change at the $i$-th symbol of the $k$-frame,

$w_{k, i}$ is the noise sample at the $i$-th symbol of the $k$-frame.

For $i=0$, the sample signal is

$$
r_{k, 0}=p_{k, 0} y_{k, 0}+w_{k, 0}
$$

where $p_{k, 0}$ is the pilot symbol in the $k$-th frame.

For $i=1,2, \ldots, L-1$, the sample signal is

$$
r_{k, i}=d_{k, i} y_{k, i}+w_{k, i}
$$

where $d_{k, i}$ is a data symbol at the $i$-th symbol of the $k$-th frame.
Since the pilot symbol $p_{k, 0}$ is known at the receiver, $y_{k, 0}$ can be obtained using Eqn. (1b) as

$$
y_{k, 0}=\frac{r_{k, 0}}{p_{k, 0}}-\frac{w_{k, 0}}{p_{k, 0}}
$$

At high SNRs, $y_{k, 0}$ can be estimated as

$$
\hat{y}_{k, 0}=\frac{r_{k, 0}}{p_{k, 0}}
$$

Estimates on the changes for the rest of the data symbols can be obtained by zeroth-order or first-order Gaussian prediction [4], i.e.,

$\hat{y}_{k, i}= \begin{cases}\hat{y}_{k, 0} & \text { Zeroth-Order Prediction } \\ \left(1-\frac{i}{L}\right) \hat{y}_{k, 0}+\left(\frac{i}{L}\right) \hat{y}_{k+1,0} & \text { First - Order Prediction }\end{cases}$

$$
\text { for } i=1,2, \ldots, L-1
$$

The estimated signal $\hat{y}_{k, i}$ is used to correct the fading effects in the received signal sample, $r_{k, i}$. After fading correction, the estimate of the data signal is

$$
\hat{r}_{k, i}=\frac{r_{k, i}}{\hat{y}_{k, i}}
$$

The resultant signal, $\hat{r}_{k, i}$ is then fed to the threshold detector (Figure 1) to produce one of the possible signal vector on the constellation $\hat{d}_{k, i}$. It is then decoded into the binary data $\hat{u}_{i}, \hat{u}_{i+1}, \hat{u}_{i+2}, \hat{u}_{i+3}$. The process repeats for all the received frames.

\section{Improved Zeroth-Order Prediction}

The technique proposed here makes use of the pilot and data symbols for multipath fading compensation. The signal $\hat{y}_{k, 0}$ is initially obtained from the pilot symbol using Eqn. (3) and is treated as the estimate of $y_{k, 1}$, i.e.,

$$
\tilde{y}_{k, 1}=\hat{y}_{k, 0}=\frac{r_{k, 0}}{p_{k, 0}}
$$

This signal is then used to correct the fading effects in $r_{k, 1}$ to give an estimate of the data signal

$$
\hat{r}_{k, 1}=\frac{r_{k, 1}}{\tilde{y}_{k, 1}}
$$

The signal $\hat{r}_{k, 1}$, again is fed to the threshold detector to produce the data signal $\hat{d}_{k, 1}$. The signal $\hat{d}_{k, 1}$ subsequently is decoded to give the binary data $\hat{u}_{i}, \hat{u}_{i+1}, \hat{u}_{i+2}, \hat{u}_{i+3}$. Since 
$r_{k, 1} / \hat{d}_{k, 1}$ is closer to $y_{k, 2}$ than $\hat{y}_{k, 0}$ in a slowly faded channel and $\hat{d}_{k, 1}$ is a possible signal vector on the constellation, an improved estimate of $y_{k, 2}$ can be obtained by

$$
\tilde{y}_{k, 2}=\frac{r_{k, 1}}{\hat{d}_{k, 1}}
$$

The signal $\tilde{y}_{k, 2}$ is used to correct the fading effects in $r_{k, 2}$. This process continues for the rest of the data symbols in the same frame according to

$$
\tilde{y}_{k, i}=\frac{r_{k, i-1}}{\hat{d}_{k, i-1}} \quad \text { for } i=2,3, \ldots, L-1
$$

The whole process repeats for every frame.

\section{Improved First-Order Prediction}

For the frame lengths of $4,8,16, \ldots$, or $2^{n}$, where $n$ is an integer, a better performance can be obtained by the improved first-order prediction using the pilot and data symbols. The proposed compensation process consists of two stages described as follows. The first stage is the compensation for the data symbols in the even-number positions (i.e., $i=2,4, \ldots, L-2$ ) of a frame. The second stage is for the data symbols in the oddnumber positions (i.e., $i=1,3, \ldots, L-1$ ) of the same frame.

\section{Compensation for Data Symbols in Even-Number Positions}

The changes on the pilot symbols in the $k$-th frame, $\hat{y}_{k, 0}$, and in the $k+1$-th frame, $\hat{y}_{k+1,0}$, can be obtained from $r_{k, 0}, p_{k, 0}$, $r_{k+1,0}$, and $p_{k+1,0}$, using Eqn. (3). The estimate of $y_{k, L / 2}$ is obtained as

$$
\hat{y}_{k, L / 2}=\frac{\hat{y}_{k, 0}+\hat{y}_{k+1,0}}{2}
$$

This signal is then used to correct the fading effects in $r_{k, L / 2}$ to give an estimate of the data signal

$$
\hat{r}_{k, L / 2}=\frac{r_{k, L / 2}}{\hat{y}_{k, L / 2}}
$$

If $L=4$, the compensation process for the data symbols in the even-number positions is completed and the compensation process for the data symbols in the odd-number positions begins.

However, if $L \geq 8$, the compensation process for the data symbols in the even-number positions continues as follows. Since $\hat{d}_{k, L / 2}$ is a possible signal vector on the constellation and $r_{k, L / 2} / \hat{d}_{k, L / 2}$ is closer to $y_{k, L / 4}$ and $y_{k, 3 L / 4}$ than $\hat{y}_{k, 0}$ and $\hat{y}_{k+1,0}$, respectively, in a slowly faded channel, the better estimates of $y_{k, L / 4}$ and $y_{k, 3 L / 4}$ can be obtained as, respectively,

$$
\begin{aligned}
& \tilde{y}_{k, L / 4}=\frac{1}{2}\left(\hat{y}_{k, 0}+\frac{r_{k, L / 2}}{\hat{d}_{k, L / 2}}\right) \\
& \tilde{y}_{k, 3 L / 4}=\frac{1}{2}\left(\frac{r_{k, L / 2}}{\hat{d}_{k, L / 2}}+\hat{y}_{k+1,0}\right)
\end{aligned}
$$

These signals $\tilde{y}_{k, L / 4}$ and $\tilde{y}_{k, 3 L / 4}$ are used to correct $r_{k, L / 4}$ and $r_{k, 3 L / 4}$, respectively, to obtain the signals

$$
\begin{gathered}
\hat{r}_{k, L / 4}=\frac{r_{k, L / 4}}{\tilde{y}_{k, L / 4}} \\
\hat{r}_{k, 3 L / 4}=\frac{r_{k, 3 L / 4}}{\tilde{y}_{k, 3 L / 4}}
\end{gathered}
$$

If $L \geq 16$, the compensation process continues further. The newly corrected signals, $\hat{d}_{k, L / 4}$ and $\hat{d}_{k, 3 L / 4}$, are used together with the pilot symbols, $p_{k, 0}$ and $p_{k+1,0}$, to correct the data symbols according to

$$
\begin{gathered}
\tilde{y}_{k, L / 8}=\frac{1}{2}\left(\hat{y}_{k, 0}+\frac{r_{k, L / 4}}{\hat{d}_{k, L / 4}}\right) \\
\tilde{y}_{k, 7 L / 8}=\frac{1}{2}\left(\frac{r_{k, L / 4}}{\hat{d}_{k, L / 4}}+\hat{y}_{k+1,0}\right) \\
\tilde{y}_{k, j L / 8}=\frac{1}{2}\left(\frac{r_{k,(j-1) L / 8}}{\hat{d}_{k,(j-1) L / 8}}+\frac{r_{k,(j+1) L / 8}}{\hat{d}_{k,(j+1) L / 8}}\right) \text { for } j=3,5
\end{gathered}
$$

Similarly, if $L \geq 32$, the compensation process continues with

$$
\begin{aligned}
& \tilde{y}_{k, L / 16}=\frac{1}{2}\left(\hat{y}_{k, 0}+\frac{r_{k, L / 8}}{\hat{d}_{k, L / 8}}\right) \\
& \tilde{y}_{k, 15 L / 16}=\frac{1}{2}\left(\frac{r_{k, L / 8}}{\hat{d}_{k, L / 8}}+\hat{y}_{k+1,0}\right) \\
& \tilde{y}_{k, j L / 16}=\frac{1}{2}\left(\frac{r_{k,(j-1) L / 16}}{\hat{d}_{k,(j-1) L / 16}}+\frac{r_{k,(j+1) L / 16}}{\hat{d}_{k,(j+1) L / 16}}\right) \\
& \text { for } j=3,5, \ldots, 13
\end{aligned}
$$

Similar equations can be derived for $L=64,128, \ldots, 2^{n}$. The compensation process continues until all the data symbols in the even-number positions are corrected. Then the compensation process for the data symbols in the odd-number positions begins as described in the next section. 


\section{Compensation for Data Symbols in Odd-Number Positions}

For compensation of the data symbols in odd-number positions, $\tilde{y}_{k, i}$ and $\hat{r}_{k, i}$ are obtained as

$$
\begin{aligned}
& \tilde{y}_{k, 1}=\frac{1}{2}\left(\hat{y}_{k, 0}+\frac{r_{k, 2}}{\hat{d}_{k, 2}}\right) \\
& \tilde{y}_{k, L-1}=\frac{1}{2}\left(\frac{r_{k, L-2}}{\hat{d}_{k, L-2}}+\hat{y}_{k+1,0}\right) \\
& \tilde{y}_{k, j}=\frac{1}{2}\left(\frac{r_{k, j-1}}{\hat{d}_{k, j-1}}+\frac{r_{k, j+1}}{\hat{d}_{k, j+1}}\right) \text { for } j=3,5, \ldots, L-3 \\
& \hat{r}_{k, i}=\frac{r_{k, i}}{\tilde{y}_{k, i}} \quad \text { for } i=1,3, \ldots, L-1
\end{aligned}
$$

Thus, all the received data symbols in even- and oddnumber positions of the frame are corrected according to Eqn. (8) to (12). The corrected signal $\left\{\hat{r}_{k, i}\right\}$ are then fed to the threshold detector and decoded into the binary data $\left\{\hat{u}_{i}\right\}$. The whole compensation process repeats for all the received frames.

\section{Results and Discussions}

Intensive Monte Carlo simulations have been carried out to evaluate the BER performance of the system in Figure 1. The signal-to-noise ratio is taken as

$$
\mathrm{SNR}=10\left[\log \left(\frac{E_{b}}{N_{0}}\right)\right] \mathrm{dB}
$$

where $E_{b}$ is the average transmitted energy per bit at the transmitter output of Figure 1 and $N_{0}$ is the one-sided power spectral density of the additive white Gaussian noise measured at the same point.

A 16QAM signal and a transmission rate of $32 \mathrm{~kb} / \mathrm{s}$ with Doppler spreads $\left(f_{D} T\right)$ of $1.25 \times 10^{-3}$ and $1.0 \times 10^{-2}$ of the symbol rate are used in all simulations. The ratio of the two BERs (obtained with and without using the data symbols) is defined as the

$$
\underset{\text { Factor }}{\text { Improvement }}=\frac{\text { BER of the system using pilot symbols only }}{\text { BER of the system using pilot and data symbols }}
$$

The results of the simulation on the BER performances of the system using improved zeroth-order prediction, with different frame lengths and with $f_{D} T=1.25 \times 10^{-3}$, are shown in Figure 3. It can be seen that, the smaller the value of $L$, the better is the performance of the system. This is more noticeable in fast fading conditions because the changes in the signals are needed to be estimated more often. At low SNRs, none of the pilot symbol-aided technique is recommended because the presence of Gaussian noise reduces the accuracy of the estimates $\tilde{y}_{k, i}, \hat{y}_{k, i}$, and $\hat{r}_{k, i}$. Figure 3 shows that, with a SNR of $45 \mathrm{~dB}$, a bit-error of about $9 \times 10^{-4}$ can be achieved even when the frame length is 64 . The improvement factors with $f_{D} T$ of $1.25 \times 10^{-3}$ and $1.0 \times 10^{-2}$ are shown in Figures 4 and 5 respectively. Clearly, the BER performance can be improved significantly by using the information in the data symbols as well as that in the pilot symbols. With $f_{D} T=1.25 \times 10^{-3}$, a SNR at $45 \mathrm{~dB}$ and frame length of 128 , the BER performance can be improved by a factor of about 46 . Whereas with $f_{D} T=1.0 \times 10^{-2}$, a SNR of $45 \mathrm{~dB}$ and a frame length of 32 , the improvement factor of about 6.8 can still be achieved.

For improved first-order prediction, the performance of the system is shown in Figure 6. Comparing the results with those of Figure 3 indicates that first-order prediction is superior. The improvement factors for first-order prediction are shown in Figure 7 and 8 . It can be seen that, with $f_{D} T=1.25 \times 10^{-3}$, a SNR at $45 \mathrm{~dB}$ and a frame length of 128 , the improvement factor is about 2 . With $f_{D} T=1.0 \times 10^{-2}$, a SNR at $45 \mathrm{~dB}$ and a frame length of 32 , the improvement factor reduces to about 1.6.

Figures 4, 5, 7 and 8 also show that the proposed zerothorder and first-order predictions begin to gain improvements when the SNR is greater than $20 \mathrm{~dB}$.

\section{Conclusions}

Multipath fading compensation techniques using the pilot symbols and data symbols for a 16QAM system have been studied using computer simulations. The results have shown that, with the use of only the pilot symbols, the system suffers a significant degradation in BER performance if the frame length is increased to 32,64 , or 128 . However, if the data symbols are used, substantial improvements in the BER performance can be obtained even with a long frame length. It has been found that, at high SNRs, and in the slowly faded channels, the BER can be reduced by a factor of about 46 and 2 using improved zerothorder and first-order prediction respectively, relative to those obtained using the pilot symbols only.

\section{References}

[1] M. L. Moher and J. H. Lodge, "TCMP-A Modulation and Coding Strategy for Rician Fading Channel," IEEE Journal on Selected Areas in Communications, vol. 7, no. 9, pp. 1347-1355, December 1989.

[2] S. Gurunathan and K. Feher, "Fade Compensation in QPRS Mobile Radio Modems," in Proceedings of the IEEE Vehicular Technology Society Conference, St. Louis, U.S.A., May 1991, pp. 144-148.

[3] J. K. Cavers, "An Analysis of Pilot Symbol Assisted Modulation for Rayleigh Fading Channels," IEEE Transactions on Vehicular Technology, vol. 40 , no. 4, pp. 686-693, November 1991

[4] S. Sampei and T. Sunaga, "Rayleigh Fading Compensation for QAM in Land Mobile Radio Communications," IEEE Transactions on Vehicular Technology, vol. 42, no. 2, pp. 137-147, May 1993.

[5] J. K. Cavers and J. Varaldi, "Cochannel Interference and Pilot Symbol Assisted Modulation," IEEE Transactions on Vehicular Technology, vol. 42, no. 4, pp. 407-413, November 1993.

[6] C. L. Liu and K. Feher, "Pilot-Symbol Aided Coherent $M$-ary PSK in Frequency-Selective Fast Rayleigh Fading Channel," IEEE Transactions on Communications, vol. 42, no. 1, pp. 54-62, January 1994.

[7] H. K. Lau and S. W. Cheung, "A Pilot Symbol-Aided Technique Used for Digital Signals in Multipath Environments, in Proceedings of the IEEE International Conference on Communications, New Orleans, U.S.A., May 1994, pp. 1126-1130.

[8] W. C. Y. Lee, Mobile Communications Engineering. New York: McGrawHill, 1983. 


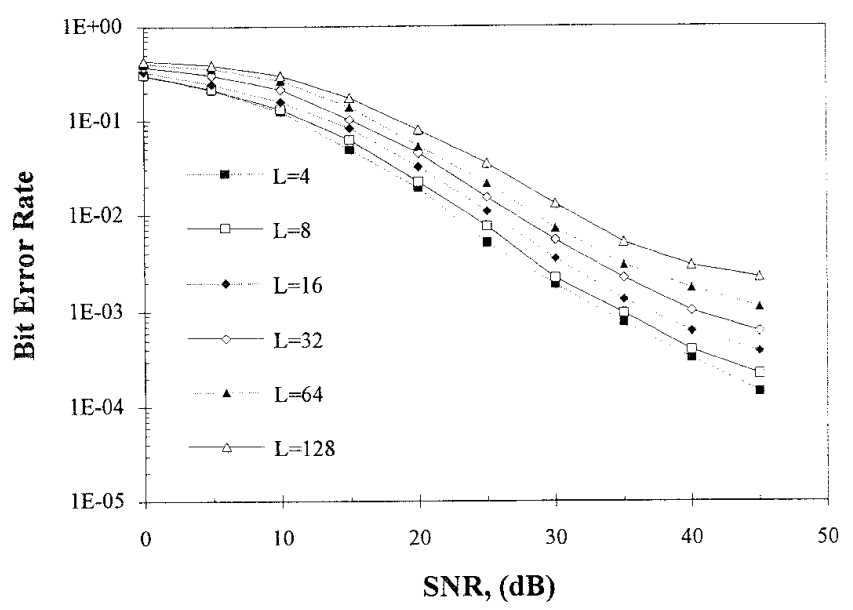

Figure 3 Performance of 16QAM Using Improved Zeroth-Order Prediction with $f_{D} T$ of $1.25 \times 10^{-3}$

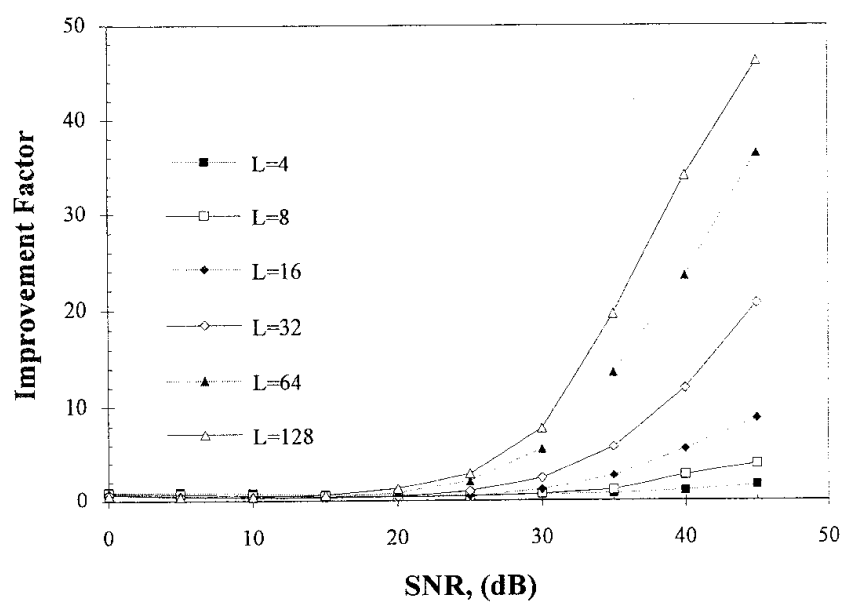

Figure 4 Improvement Factor of Improved Zeroth-Order Prediction with $f_{D} T$ of $1.25 \times 10^{-3}$

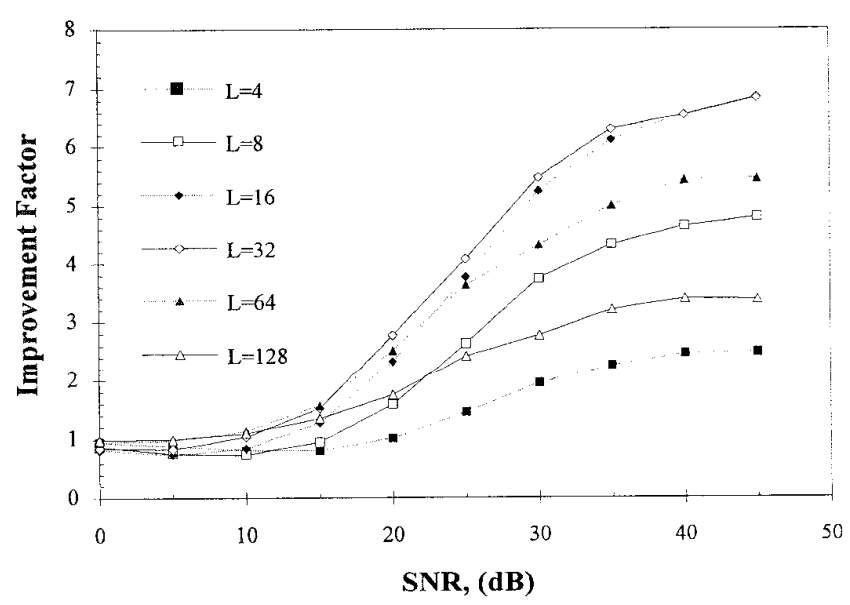

Figure 5 Improvement Factor of Improved Zeroth-Order Prediction with $f_{D} T$ of $1.0 \times 10^{-2}$

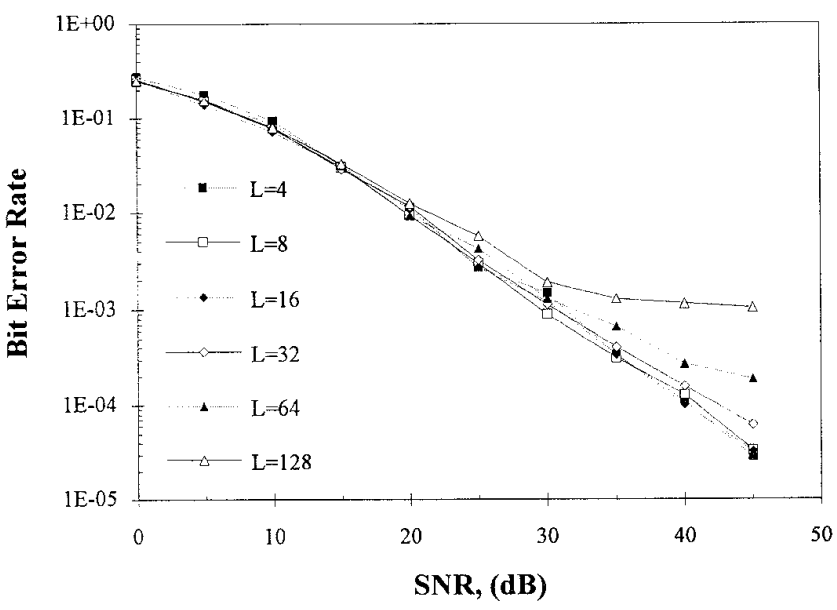

Figure 6 Performance of $16 Q A M$ Using Improved First-Order Prediction with $f_{D} T$ of $1.25 \times 10^{-3}$

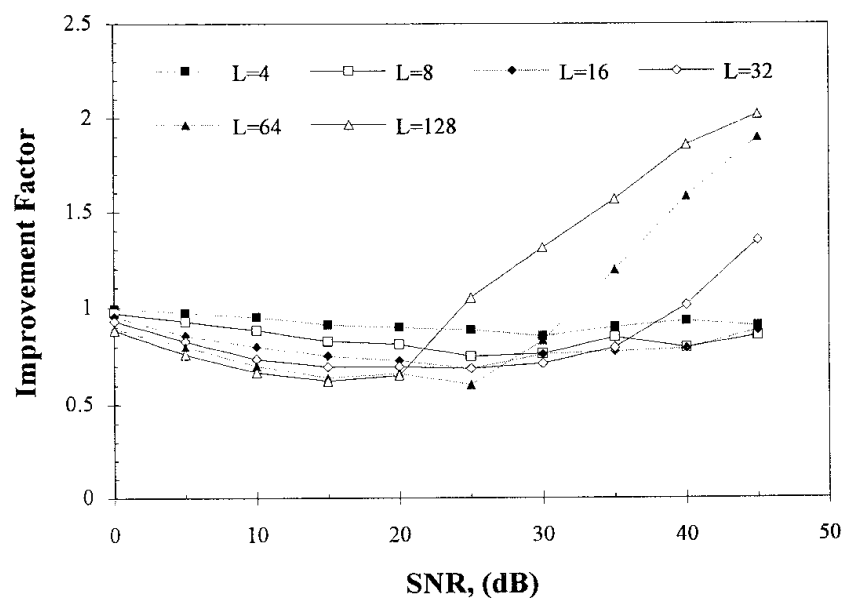

Figure 7 Improvement Factor of Improved First-Order Prediction with $f_{D} T$ of $1.25 \times 10^{-3}$

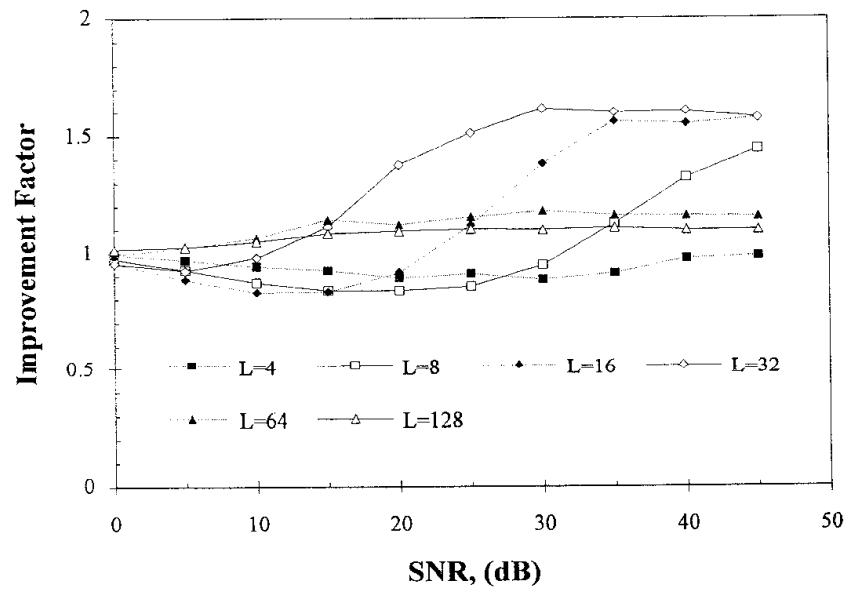

Figure 8 Improvement Factor of Improved First-Order Prediction with $f_{D} T$ of $1.0 \times 10^{-2}$ 